\title{
Communication citoyenne et coopération interculturelle
}

\section{Christian Mesnil}

\section{(2) OpenEdition \\ 12 Journals}

Édition électronique

URL : http://journals.openedition.org/communicationorganisation/2838

DOI : 10.4000/communicationorganisation.2838

ISSN : $1775-3546$

Éditeur

Presses universitaires de Bordeaux

Édition imprimée

Date de publication : 1 mai 2003

ISSN : 1168-5549

Référence électronique

Christian Mesnil, "Communication citoyenne et coopération interculturelle », Communication et organisation [En ligne], 23 | 2003, mis en ligne le 27 mars 2012, consulté le 19 avril 2019. URL : http:// journals.openedition.org/communicationorganisation/2838; DOI : 10.4000/ communicationorganisation.2838

Ce document a été généré automatiquement le 19 avril 2019

(c) Presses universitaires de Bordeaux 


\title{
Communication citoyenne et coopération interculturelle
}

\author{
Christian Mesnil
}

1 Depuis les temps immémoriaux (1,7 million d'années?) où les premiers hominidés ont quitté le berceau africain de l'humanité pour passer en Europe et en Asie par « le couloir du levant ", l'actuel proche orient, les vagues migratoires n'ont jamais cessé. L'histoire de l'évolution humaine est profondément liée à cette notion de mouvement dont Pascal faisait une définition même de la vie par opposition au « repos entier de la mort ». Qu'ils franchissent ou non des frontières, les hommes sont constamment amenés pour des raisons diverses à se déplacer soit pour s'intégrer dans un milieu ou une organisation inconnus, soit pour accueillir et faire de la place. Partager, marquer ou conquérir un nouveau territoire, s'organiser des sécurités n'est facile pour personne. A cela s'ajoute l'adaptation mentale vis à vis d'un environnement humain, des horaires, des procédures, des modes de pensée, eux aussi différents et en évolution.

Dans ce domaine, les universités semblent occuper une place privilégiée pour favoriser l'apprentissage du respect de l'autre, les mises en situation de communication voire d'actions, de coopération, de solidarité. D'une part, les étudiants (comme les enseignants) doivent s'insérer dans une société plus hétérogène et travailler dans ou avec des organisations internationales parfois sur des sites délocalisés ou avec des collaborateurs étrangers. D'autre part, ayant atteint leur majorité, les étudiants sont à une époque charnière qui favorise leurs questionnements, leur autonomie et leur prise de responsabilité.

Des échanges, des stages, des projets internationaux de coopération initient à la prise de décision individuelle et ou collective. Ils constituent les vecteurs privilégiés d'une construction identitaire et d'une consolidation de citoyenneté non limitée à un pays ou continent. Ces travaux pratiques en quelque sorte qui se déroulent non en laboratoire ou salle de cours mais sur le terrain de la réalité nécessitent un certain nombre de compétences parmi lesquelles la capacité à communiquer est fondamentale. 
4 La présente réflexion tente d'aborder cinq aspects déterminants pour communiquer et coopérer de façon citoyenne. Sont envisagés successivement l'hétérogénéité des contextes, les enjeux matériels, la compétence des acteurs, la médiatisation de la coopération et le paradoxe d'un contrat de projet.

\section{Différences et convergences du contexte}

Le rapprochement de deux éléments contrastés n'est pas qu'un procédé rhétorique ou artistique classique pour contribuer à la mise en valeur réciproque d'éléments simplement juxtaposés. C'est à partir du moment où les acteurs baignent dans des contextes différents (histoire, climat, paysage, urbanisme, organisation sociale, pratique religieuse, économie, alimentation, codes vestimentaires, etc.) qu'il y a matière à échange et confrontation ${ }^{1}$.

6 Plus ces différences sont importantes qualitativement et quantitativement, plus les sources de questionnements seront nombreuses et variées. La gamme de sujets potentiels de coopération devient de ce fait très riche. De plus l'absence d'enjeux en termes de concurrence devrait alors faciliter l'objectivité, la neutralité dans le choix et la conduite des échanges qui valorisent en fait chacun des partenaires. La recherche d'identité passe par l'autre qui nous nomme, nous interroge et nous situe. L'échange ne peut se faire qu'entre des milieux hétérogènes. Dans de nombreux domaines les scientifiques constatent l'existence de territoires entre lesquels il n'est pas nécessaire d'aboutir toujours à un consensus flou, ambigu. Une situation de communication vraie n'est préservée qu'à partir du maintien des spécificités de chacun (artistique, scientifique...)

7 Pourtant, si le découpage de la réalité, les normes sociales, les pratiques et les mythes sont par trop éloignés d'une société à l'autre, l'échange devient fragile et aléatoire ${ }^{2}$. En outre, chacun de nous n'accepte d'être interpellé par des comportements ou convictions différents que s'il a par ailleurs plus ou moins consciemment l'assurance de préserver un certain équilibre ou de retrouver une certaine stabilité par rapport à ses propres valeurs. Enfin, le fait de pouvoir se tourner vers des procédures, des préoccupations ou des convictions communes crée des points d'ancrage, des zones de références partagées, des temps de complicité et de reconnaissance mutuelle. Sur le plan universitaire par exemple, l'intérêt commun peut résider dans une technologie, un cursus de formation, une procédure expérimentale, une discipline ou un auteur. De ce fait, l'appartenance à un même réseau identifiable par des préoccupations, des rythmes, des espaces de rencontre proches ou complémentaires constitue un atout indéniable, que l'existence de ce réseau soit officielle ou informelle.

8 Finalement la qualité d'un échange semble curieusement reposer sur un équilibre fragile entre des différences qui nourrissent et justifient le dialogue et des points communs ou convergences sources de confiance et de solidarité. La précarité de l'équilibre tient au fait que cette tension permanente n'est jamais statique. Constamment, des disparités s'estompent, d'autres se maintiennent ou s'accroissent. Si elles deviennent divergences massives ou rédhibitoires et que les espaces d'actions ou de convictions proches ou partagées ne sont pas assez solides, l'échange risque alors d'être ébranlé voire interrompu. 


\section{Communiquer sur des enjeux matériels}

9 Tout échange suppose pour être mis en œuvre des moyens humains, matériels et/ou financiers. Trouver des fonds pour une action peut constituer un formidable outil de communication, d'information et de sensibilisation. Au lieu d'une démarche d'assistanat, s'instaure alors une mobilisation, source de motivation, de solidarité et d'engagement. Parfois, le projet ponctuel et/ou localisé devient un échange de plus grande envergure dans l'espace et le temps. Il convient dès lors de mettre en place un réseau structuré et/ ou de s'adresser à une organisation existante qui facilitera l'accès à des financements, des aides matérielles ou humaines.

Le cheminement logique de la micro réalisation à la mise en place ou utilisation d'une organisation plus ou moins lourde à gérer conduit à des dérives classiques. L'essentiel de la communication peut alors se trouver investi dans la recherche de moyens au détriment d'un projet véritablement porteur, personnalisé, partagé. Dans d'autres cas les efforts déployés tiennent de l'habileté procédurale ou communicationnelle, du lobbying pour présenter des dossiers efficaces, les faire soutenir par les bons interlocuteurs, afin de décrocher les financements. De là à lier l'efficacité d'une communication à l'importance des moyens mis en œuvre, à la professionnalisation de celle-ci ou à l'exceptionnel de l'événement mis en place, il n'y a qu'un pas.

11 Entre le souci légitime de maintenir une organisation qui facilite, structure, permet la continuité et l'entrée dans un système qui pérennise, fonctionnarise, la limite est particulièrement ténue. Lorsque le financement prime avec prélèvements plus ou moins justifiés aux différents échelons de l'organisation, l'échange se fragilise ${ }^{3}$. Des acteurs peuvent parfois se sentir utilisés voire exploités et se démotivent. L'enjeu du projet se déplace vers des préoccupations financières ou de pouvoir, personnelles ou collectives, avec des stratégies et critères de la société marchande (par exemple : le plus offrant ou la sélection par l'argent.)

Être dégagé d'un certain nombre de contingences matérielles, ne pas y perdre financièrement, voir reconnu et indemnisé légitimement le temps passé à un travail de qualité qui aurait été plus rémunérateur financièrement dans un autre contexte, certes, mais si les moyens priment sur la fin, ils risquent inexorablement d'occuper peu à peu l'essentiel des messages transmis. L'objet de la communication se déplace vers des informations organisationnelles ou financières qui en viennent à occulter le projet. Progressivement la priorité est accordée à une communication technique, statistique, comptable qui en fait se contente de justifier, classer, répertorier, (dé)montrer. Toute la gamme d'attitudes conscientes ou inconscientes est alors possible entre le silence absolu sur les sujets tabous, la transparence totale où il faut rendre compte de tout dans les moindres détails en passant par une opacité variable suivant les sujets, les époques et les protagonistes. Avec ou sans préoccupations institutionnelles, la communication semble constamment menacée de dérives liées à des enjeux matériels, financiers ou humains. Le double langage voire la langue de bois guettent à tout moment les plus vigilants et les petites ou moyennes organisations de la société civile impliquées dans des problématiques de citoyenneté ou de coopération n'en sont pas plus exemptes que les autres. Entre le professionnalisme du contrôle et la générosité de la confiance, entre la nécessité d'un certain retour sur investissement et la solidarité d'un don par nature vraiment gratuit, désintéressé et sans arrière pensée, une veille, un arbitrage régulier 
sont indispensables. Dès lors l'émission d'un message clair mais non réducteur ou naiff semble toujours difficile. La prudence et le provisoire doivent souvent tempérer la générosité et l'enthousiasme dans une parole qui pour être vraiment citoyenne et crédible paraît condamnée à être perpétuellement sur le qui-vive.

\section{Compétences et incomplétudes des acteurs}

13 Plusieurs sciences humaines (psychologie, psychanalyse, philosophie ${ }^{4}$ ) ont souligné la présence en chacun de nous sinon de zones d'ombre, du moins de contradictions ou tensions dont nous n'avons pas toujours conscience. Avant de rencontrer l'autre, chacun devrait avoir une notion relativement précise de sa propre identité, de ses compétences et de ses motivations même si celles-ci ne sont jamais définitives. Être à peu près au clair avec sa communauté d'appartenance, son savoir, ses domaines de performances et ses limites du moment semble indispensable face à tout interlocuteur (celui qui attend une expertise, un diagnostique, un conseil comme celui qui doit répondre à un besoin, à une demande). Est-ce toujours le cas?

Par ailleurs, la capacité à communiquer de celui qui est en position d'expertise semble fondamentale. Pour cela, la langue n'est pas l'obstacle majeur parce qu'on trouve toujours une solution lorsque les partenaires sont motivés pour se comprendre mais elle n'est pas neutre car celui qui impose sa langue impose sa culture et sa vision du monde. En outre, au delà des formulations précises d'un savoir ou d'un programme détaillé et de leur décodage sans trop de malentendu, se pose la question d'une véritable intercompréhension, c'est à dire de l'aptitude à se mettre à la place de l'autre et à intégrer son raisonnement. Parfois s'ajoute à la difficulté d'un mode de pensée, d'un cheminement différent, la situation (classique chez les informaticiens) d'un client utilisateur ou demandeur qui formule mal sa requête et ne sait guère définir clairement son cahier des charges et ce qu'il attend du spécialiste.

Il ne peut exister de communication véritable sans cette écoute initiale attentive dont Cari Rogers faisait un préalable fondamental ${ }^{5}$. Parfois, nous sommes tellement habités par le projet qui nous anime, par ce que nous désirons apporter à l'autre, qu'il n'y a plus dialogue mais monologue. Le désir de transmettre ce que nous avons reçu, notre enthousiasme, nos convictions semble légitimement constituer notre préoccupation majeure. Au lieu de commencer par entendre les autres et d'intégrer véritablement leurs recherches, leurs demandes, leurs préoccupations, nous acceptons souvent mal de modifier de façon significative nos plans, nos programmes. En communication, la limite entre influence et manipulation est le libre arbitre. Quelque soit le locuteur (parent, enseignant, expert, journaliste, homme politique), il recherche à faire entrer son vis à vis dans un certain cadre, une forme de pensée. Reste à savoir si l'interlocuteur garde une liberté qu'il utilisera le moment venu'. Lorsque nous imposons plus ou moins une parole ou un projet en manipulant, en mettant en scène ou en utilisant les procédés classiques de l'argumentation-persuasion, nous nous posons comme efficace et important. Il n'y a plus d'échange, de réciprocité et de débat, le partenaire n'est pas accueilli tel qu'il est mais tel qu'on voudrait qu'il soit. La communication véritable disparaît au profit d'une prestation ou au mieux d'un échange de service.

Lorsque la compétence n'est plus que certitudes, savoirs, savoirs faire ou procédures incontournables, la communication est menacée. Le repli sur les réponses toutes faites, les habitudes, des identités guette. Parfois la compétence passe curieusement par une 
capacité à faire le vide en soi et reconnaître son incomplétude pour mieux intégrer les préoccupations de son vis à vis. Rebondir sur des territoires, des idées, des perspectives inexplorées devient alors possible.

\section{Médiatisation de l'action}

17 Dans la gestion d'un projet, nous rêvons tous de temps forts, de la performance d'une programmation efficace, d'une coopération dynamique, maitrisée, fructueuse, quantitativement et qualitativement. A cette obsession du résultat peut s'ajouter le souci de faire connaître qui valorise nos actions et la recherche de l'influence sociale et médiatique qui en découle. Parfois, cette communication devient par surcroît une mise en spectacle involontaire imposée par le système médiatique ${ }^{7}$.

Cette prétention à tout maîtriser et à le faire savoir haut et fort peut conduire à un activisme qui épuise ou à une marche forcée qui use les plus faibles ou suscite des résistances. Des moments intenses et exceptionnels n'ont de sens que s'ils sont programmés de façon intermittente et non dans un esprit de surenchère, de compétition, de spectacle ou de représentation. Accepter d'agir parfois dans une relative discrétion, ou communiquer sans avoir pour critère le nombre d'interlocuteurs concerné ou l'importance de l'événement constituent des attitudes qui construisent une relation parfois plus solide.

Par ailleurs les rencontres ordinaires, non médiatiques ne sont pas à rater, les occasions banales, quotidiennes, utilitaires qui paraissent dérisoires sont peut être plus importantes que l'urgent et l'essentiel des professionnels du management. Nos réflexes de gains de temps et de flux tendus nous amènent peut être à brûler les étapes, forcer les mentalités et déclencher des résistances alors que la notion de temps qui passe est relative à des contextes, des modes de vies et des procédures différentes selon les cultures.

De plus, tâtonner, tester, procéder par essai en bricolant modestement une solution provisoire permet de limiter les déconvenues et ouvre à nouveau le droit à l'erreur et la possibilité d'échec. Pour maintenir un niveau de performance déterminant dans notre quête de réussite, tout ce qui n'est pas événement, exception, record devient suspect. On parle alors de " has been », de stagnation, de déclin ou de régression. Pour communiquer sur leurs échecs, leurs limites, leurs stress, l'individu comme l'organisation restent particulièrement démunis et maladroits voire durs et injustes. La culpabilisation, l'incompétence, l'impuissance, l'inconscience: les jugements péremptoires, les connotations péjoratives, implicites ou explicites surviennent vite. L'ouvrage de MarieFrance Hirigoyen intitulé Le Harcèlement moral montre combien les conséquences de l'échec ou de la non performance sont démultipliées par une communication interpersonnelle qui peut devenir humiliante et destructrice ${ }^{8}$.

Dans certains cas, l'efficacité doit pouvoir passer par des temps de pause, de respiration et de ressourcement. Au lieu d'une marche forcée, d'une fuite en avant, laisser du temps au temps et ménager des espaces de silence ou de communication allégée constitue souvent un gage de prudence et de ténacité. Mettre un projet en sommeil ou faire tourner une collaboration au ralenti ne signifie pas forcément y renoncer. Or nous sommes marqués par une société où le travail constitue une valeur et la non activité comme la non communication paraît pour le moins suspecte (incompétence, paresse, dissimulation.) Dans la société romaine, il en allait autrement car le citoyen se devait de garder l'esprit 
libre pour veiller et participer aux affaires de la cité. Peut-être y a-t-il actuellement d'autres cultures qui gardent plus ou moins cette conception et qui souhaiteraient un rythme différent de coopération?

En outre, nos actions sont constamment accompagnées d'informations ou communications qui, lorsqu'elles s'estompent, laissent un grand vide, donnent l'impression de ne plus exister ou de céder la place à des organisations concurrentes. Gérer des temps de silence qui ne soient pas une disparition de la non communication, un refus d'échanger, un éloignement progressif précurseur d'indifférence, constitue une pratique délicate. Entre le silence diplomatique préférable à des paroles blessantes, le silence synonyme de manque de courage, le silence prémédité du teasing publicitaire, le silence contraint du plus vulnérable et le silence librement consenti, partagé entre des acteurs complices, confiants et solidaires, s'intercale toute une palette de nuances et de silences intermédiaires.

Que faut-il faire? Communiquer pour ne rien dire, pour exister et meubler un silence de plus en plus dépaysant dans le brouhaha médiatique qui nous environne ou échanger par des actes tranquilles qui, même modestes, sont parfois plus parlants? Mais nous savons tous combien les crises et conflits sont inhérents à la coopération humaine. Même imparfaite la communication se révèle parfois indispensable en essayant de discerner les contenus, les moments et les formulations les moins inadaptés ${ }^{10}$.

\section{La confiance et le contrat}

Depuis vingt ans la démarche de management de projet ne cesse de s'affirmer comme une réponse opérationnelle dans les situations d'organisation et de coopération. Alors que la pratique d'un métier entraîne le plus souvent des contraintes, des règles, des structures imposées par un professionnel expérimenté dans le cadre d'une production souvent massifiée et standardisée, la gestion de projet fonctionne autrement. Il s'agit de coordonner différentes actions au sein d'une démarche spécifique, non répétitive, en fonction d'un but. Ses caractéristiques principales sont des décisions irréversibles, de l'incertitude forte (risques financiers, techniques, humains) et des variables exogènes (climat, concurrence, évolution des besoins du partenaire etc.) La structure mise en place devient temporaire, non hiérarchique, avec des responsabilités horizontales en interdépendance et un raisonnement bâti sur des hypothèses et du métissage.

Cette démarche d'action a inévitablement entraîné une modification des processus communicationnels que Gino Gramaccia ${ }^{11}$ identifie notamment en deux pôles. D'une part une communication connectique plus technique, constituée d'ajustements, de coordination, de flexibilité sur des segments très courts avec des acteurs parfois éloignés. Il s'agit de transmettre des informations vitales, précises à des destinataires bien identifiés sans oublier de normaliser ensuite et d'archiver pour pouvoir réutiliser. D'autre part, une communication fiduciaire, de confiance, un engagement réciproque d'acteurs marqués parfois par la distance sociale, professionnelle, géographique, culturelle. Mais il s'agit bien d'un engagement qui doit être clairement dit, sans silence ni omission, et donc contractualisé.

Dans cette juxtaposition confiance/contrat semble résider un autre paradoxe caractéristique des rapports humains et de la dichotomie implicite/explicite présente dans tout échange. La confiance se mérite, se tisse peu à peu au fil du temps en opérant 
notamment un maillage entre les compétences, le professionnalisme des partenaires. La confiance repose sur la notion même d'équipe et sur une connectique efficace, médiatisée ou non. Pourtant quelle que soit la performance des outils technologiques, quelles que soient la compétence et la polyvalence des acteurs, l'échange a besoin pour exister vraiment d'une relation interpersonnelle à chaque fois unique, recommencée et imprévisible. Si les papiers, téléphones, messages internet ne sont pas complétés de rencontres directes, la communication risque de ne se limiter qu'à des mots, des formulations et du savoir. Si au nom du pragmatisme organisationnel, les hommes sont purement interchangeables comme des pièces de machine, ou le poids de l'équipe et de la connectique tel que la liberté de l'individu mandaté n'existe plus, la rencontre véritable disparait. La confiance repose autant sur du rationnel que sur du relationnel qui ne se décrète pas, ne s'institutionnalise pas par contrat. Mais à l'inverse dans la construction de sens, de plaisir partagé, de motivation, la liberté ne peut être absolue. Le contrat est indispensable pour marquer des limites, rappeler des frontières entre par exemple la coopération, la complicité et le copinage. Le contrat concerne l'autre, étranger ou non, qui est par définition inattendu, envahissant parfois, et peut faire tout à coup irruption dans un déroulement de projet au risque de l'infléchir complètement. De plus, le contrat concerne aussi l'autre moi-même qui se révèle dans l'action différent de ce qu'il pensait être ou dont l'environnement peut se modifier ou dont la motivation peut s'infléchir. La nécessité d'un contrat citoyen établi et géré avec loyauté semble bien par conséquent le contrepoint nécessaire d'une confiance, d'un optimisme et d'un plaisir sans lequel une coopération n'a pas beaucoup de sens. Mais l'un et l'autre restent d'une grande fragilité. Parfois, c'est une maladresse, une erreur ou un découragement qui est à l'origine d'une réorientation de l'action ou d'un succès inattendu. Michel Crozier et Ehrard Friedberg voici un quart de siècle soulignaient déjà combien la rationalité est limitée et contingente ${ }^{12}$. Tout ne peut être dit et contractualisé, l'événement essentiel survient parfois de façon imprévisible pour bouleverser nos plans, nos programmes, nos sécurités ou nos contrats.

\section{Dialoguer et rester constamment en état de veille}

Dans les processus de coopération, la transmission d'un savoir ou savoir faire s'accompagne progressivement d'une complexité grandissante. D'une part le contenu du message ainsi que les compétences sont de plus en plus mosaïques et provisoires. D'autre part, les interlocuteurs sont de plus en plus variés, éloignés culturellement ou spécialisés dans des procédures ou disciplines sophistiquées. Parasité par des enjeux de pouvoir, de notoriété ou de reconnaissance, chacun se révèle finalement producteur d'énigmes au moins aussi nombreuses que les informations qu'il formule ou les connaissances qu'il découvre.

La célèbre maxime de Descartes: "Je me donne pour règle de ne jamais considérer comme certain ce qui peut paraitre indubitable » n'est elle pas toujours d'actualité et ne risque-t-elle pas d'aboutir alors à un scepticisme paralysant, un pessimisme chronique voire à du non sens, de l'absurde, du découragement, du désespoir? Dans de nombreux domaines (scientifiques, économiques, communicationnels...) les spécialistes ${ }^{13}$ constatent la présence de systèmes chaotiques qu'ils distinguent soigneusement des mouvements anarchiques car il est possible au moins de les modéliser après coup. Par ailleurs la pratique du véritable dialogue, c'est-à-dire de phases de questionnement, de remise en 
cause, de propositions passe de plus en plus pour la situation de communication la moins inadaptée parce que sensible à la transformation de la situation initiale.

A la suite peut-être d'Habermas ${ }^{14}$, cet optimiste du dialogue, et de ses travaux sur l'éthique de la discussion, ont été soulignés au moins deux atouts irremplaçables de l'échange entre des milieux hétérogènes: la variété des registres possibles et l'imprévisibilité du dialogue interactif (direct ou médiatisé).

Le dialogue devient ainsi progressivement un outil incontournable dans les processus de médiation et de gouvernance qui accompagnent de plus en plus les démarches de coopération. A tout citoyen de notre planète incombe idéalement la responsabilité de s'informer, de communiquer, d'essayer de comprendre et respecter puis d'agir individuellement ou collectivement en conformité avec sa conscience. Certes tous ne peuvent pas prendre part à ce dialogue mais ceux qui ont la chance d'y participer ou de le nourrir doivent constamment rester en état de veille, assortir leurs décisions et enthousiasmes de prudence, de vigilance et de modestie.

31 Car « l'expression du désir de donner du sens à la vie dans ce qu'elle a de plus beau et de plus énigmatique à la fois, ne sera jamais le résultat mécanique de liaison hypertextuelles. Cette expression est d'un autre ordre. Elle relève de l'intériorité, comprise comme ce qui constitue la personne et qui ne peut être complètement extériorisé ou modélisé, et qui par conséquent échappe à toute maitrise et renvoie à cette part de l'homme qui reste mystérieuse et qui est source vive de toute création ${ }^{15}$.»

\section{BIBLIOGRAPHIE}

BENOIT D. La manipulation dans la communication in Communication et Organisation ${ }^{\circ} 1,1^{\mathrm{er}}$ semestre 1998.

CLAVAL P. La géographie culturelle. Une nouvelle approche des sociétés et des milieux, 1995. rééd. Armand Colin, col. U. 2002.

CORDIER A. Le livre numérique internet et la pensée in Communication et langage $n^{\circ} 122.4^{\text {ème }}$ trim $99 \mathrm{p} 16$.

CROZIER M. et FRIEDBERG E. L'acteur et le Système, les contraintes de l'action collective, 1977, rééd. Seuil. Col. « Points essais », 1992. DEMORGON J. Complexité des cultures et de Unter culturel. Anthropos 1996.

DUCCINI H. La télévision et ses mises en scène, Nathan Université 1998. HABERMAS J. Théorie de l'agir communicationnel, 1981, trad, fr 1987 Fayard.

HIRIGOYEN M-F. Le harcèlement moral, édit. La Découverte, Syros, 1998. LAZAUBON C, GRAMACCIA G, MASSART G. Management de projet technique. Ellipse, 1998.

MARCINIAK R. Communication et Gestion des conflits dans les projets in Communication et Organisation $n^{\circ} 13.1^{\mathrm{er}}$ semestre 98.

MEDA D. Qu'est-ce que la richesse ? Flammarion, 2000. 
RICOEUR P. Soi-même comme un autre, 1990, rééd. Seuil, col. « Points essais », 1997.

ROGERS C. Le développement de la personne, rééd. Dunod, col. « Psychologie sociale », 1998.

ROSNAY (de) J. L homme symbiotique, Seuil 1995.

WIEVIERKA M. (sous la direction de) Une société fragmentée ? La découverte, 1997

\section{NOTES}

1. Claval Paul. La géographie culturelle. Une nouvelle approche des sociétés et des milieux. 1995. rééd. Armand Colin. coll.U. 2002.

2. Cf. à ce sujet l'ouvrage collectif dirigé par Michel Wievierka. Une société fragmentée ''La découverte. 1997.

3. Cf. à ce sujet le regard critique porté par Dominique Meda dans son ouvrage. Ou 'est-ce que la richesse'? (Flammarion. 2000).

4. Notamment Ricoeur Paul dans. Soi-même comme un autre. 1990. rééd. Seuil, coll. "Points essais ». 1997.

5. Rogers Carl. Le Développement de la personne, rééd. Dunod. coll. « Psychologie sociale ». 1998

6. Benoît Denis. La manipulation dans la communication in Communication et Organisation $\mathrm{n}^{\circ} \mathrm{I}$. $\mathrm{I}^{\mathrm{e}}$ semestre 1998.

7. Duccini Hélène. La télévision et ses mises en scène. Nathan Université 1998.

8. Hirigoyen Marie France. Le Harcèlement moral, édit. La Découverte et Syros. 1998.

9. Demorgon Jacques. Complexité des cultures et de l'interculturel. Anthropos 1996.

10. Marciniak Rolande. Communication et Gestion des conflits dans les projets in Communication et Organisation $\mathrm{n}^{\circ} 13.1^{\mathrm{er}}$ semestre 98.

11. Cf Lazaubon Christian. Gramaccia Gino. Massart Gerard. Management de projet technique. Ellipse 1998

12. Crozier Michel et Friedberg Ehrard: L'acteur et le système, les contraintes de l'action collective. 1977. rééd. Seuil, coll. « Points essais ». 1992.

13. Rosnay (de) Joel. L'homme symbiotique. Seuil 1995

14. Habermas Jürgen. Theorie de l'agir communicationnel. 1981. trad. fr 1987 Fayard.

15. Alain Cordier. Le livre numérique internet et la pensée, in Communication et langage $n^{\circ} 122.4^{\mathrm{e}}$ trim 99, p.16.

\section{RÉSUMÉS}

Des tensions, inhérentes à toute coopération qui se veut citoyenne, se trouvent déjà avivées lorsqu'il y a passage à une action commune. Quand cette coopération intervient dans un contexte interculturel, ces tensions semblent devoir être prises en compte avec plus de soin encore pour ne pas entraver mais au contraire dynamiser les processus notamment en ce qui concerne l'hétérogénéité des contextes, les enjeux matériels, la compétence des acteurs, la médiatisation de la coopération et la gestion de projet. 
Tensions are inherent to any kind of cooperation involving the commonalty, hut they are enhanced when a common action is undertaken. When that cooperation occurs in an intercultural context, those tensions must to he taken into account even more carefully in order not to hinder the processes hut on the contrary to make them more dynamic, especially as far as the diversity of the contexts, the material stakes, the agents'skills, the way the media are handled and the project management are concerned.

INDEX

Mots-clés : communication, interculturel, coopération, tension, veille

\section{AUTEUR}

\section{CHRISTIAN MESNIL}

Christian Mesnil est maître de conférences en sciences de l'information et de la communication à l'Université du Littoral Côte d'Opale (Calais). 